\title{
Neural interactions in unilateral colliculus and between bilateral colliculi modulate auditory signal processing
}

\author{
Hui-Xian Mei, ${ }^{1,2}$, Liang Cheng ${ }^{1}$ and Qi-Cai Chen ${ }^{1}$ * \\ ${ }^{1}$ School of Life Sciences and Hubei Key Lab of Genetic Regulation and Integrative Biology, Central China Normal University, Wuhan, China \\ ${ }^{2}$ School of Sport, Hubei University, Wuhan, China
}

\section{Edited by:}

Charles F. Stevens, The Salk Institute for Biological Studies, USA

\section{Reviewed by:}

Alex Koulakov, Cold Spring Harbor Laboratory, USA

Ken K. Yung, Hong Kong Baptist

University, China

\section{*Correspondence:}

Qi-Cai Chen, School of Life Science, Lab of Neurobiology, Central China Normal University, Luoyu Avenue 152, Wuhan 430079, Hubei, China.

e-mail:qcchen2003@yahoo.com.cn
In the auditory pathway, the inferior colliculus (IC) is a major center for temporal and spectral integration of auditory information. There are widespread neural interactions in unilateral (one) IC and between bilateral (two) ICs that could modulate auditory signal processing such as the amplitude and frequency selectivity of IC neurons. These neural interactions are either inhibitory or excitatory, and are mostly mediated by $\gamma$-aminobutyric acid (GABA) and glutamate, respectively. However, the majority of interactions are inhibitory while excitatory interactions are in the minority. Such unbalanced properties between excitatory and inhibitory projections have an important role in the formation of unilateral auditory dominance and sound location, and the neural interaction in one IC and between two ICs provide an adjustable and plastic modulation pattern for auditory signal processing.

Keywords: inferior collicular neurons, excitatory interaction, inhibitory interaction, bilateral collicular interaction, auditory signal processing

\section{INTRODUCTION}

In sound reception, auditory signal processing has traditionally been explained by neural interactions of divergent and convergent projections within the ascending auditory system through the interplay between excitation and inhibition (Suga, 1997). Auditory interactions can be found between neurons in one auditory nucleus, bilateral symmetrical auditory structures or nuclei, and even in auditory and non-auditory structures. This implies a neural modulation that plays an important role in maintaining the diversity and accuracy of auditory functions (Mei and Chen, 2010). For example, all sound signals in the range of audible frequency can be perceived by ear, however, we only notice those sounds interested by us and other sound signals that assumed to have no biological significance are filtered by excitatory or inhibitory modulation during transmission upward to different auditory nucleus.

Inferior colliculi (ICs), paired auditory structures, are located between the lower brainstem auditory nuclei and the auditory thalamus in the central auditory pathway. IC receives excitatory and inhibitory inputs from many lower auditory nuclei (Adams, 1979; Adams and Wenthold, 1979; Brunso-Bechtold et al., 1981; Adams and Mugnaini, 1984; Pollak and Casseday, 1989; Covey and Casseday, 1995; Casseday and Covey, 1996), contralateral IC (Malmierca et al., 1995, 2009) and from the primary auditory cortex (AC; Games and Winer, 1988; Herbert et al., 1991; Ojima, 1994; Saldaña et al., 1996; Malmierca and Ryugo, 2011). IC functions as an important relay station, and not only analyzes and integrates sound signals in terms of amplitude, frequency, and time course, etc., from different sources, but also prepares to route these signals to higher level center (Casseday et al., 1994; Jen et al., 1998; Suga et al., 1998; Jen and Zhang, 2000; LeBeau et al., 2001). A number of studies have shown that auditory signal processing and integration in ICs are significantly modulated by the massive descending corticofugal system which adjusts and improves ongoing collicular signal processing in multipleparametric domains but also reorganizes collicular auditory maps according to the acoustic experience (Jen et al., 1998; Jen and Zhou, 2003; Popelar et al., 2003; Yan et al., 2005; Zhou and Jen, 2007; Ma and Suga, 2008; Suga, 2008; Suga et al., 2010). However, few studies have characterized how neural circuits in or between ICs can affect collicular auditory signal processing and integration. Therefore, in this article, we review recent findings and focus mainly on neural interactions either in one IC or between two ICs.

\section{EFFECT OF INTERACTIONS BETWEEN NEURONS IN ONE IC IN THE AUDITORY SIGNAL PROCESSING}

There are extensive intrinsic connections between neurons in one IC such that the IC neurons are likely to be a major source of inputs to other IC cells (Saldaña and Merchán, 1992). Such intercollicular fibers contribute to the formation of the known fibrodendritic laminae in one IC (Herrera et al., 1988, 1989; Oliver and Schneiderman, 1991). How do the neurons inside one auditory center interact with each other? Little is known about this interaction, but immunocytochemical localization demonstrated that one IC contained considerable amounts of glutamic acid, glycine, and glutamate decarboxylase (GAD), an enzyme that catalyzed the decarboxylation of glutamate to $\gamma$-aminobutyric acid (GABA), although some of these molecules could have an extrinsic origin (Adams and Wenthold, 1979; Ottersen and StormMathisen, 1984; Vetter and Mugnaini, 1984; Moore and Moore, 1987; Roberts and Ribak, 1987; Caspary et al., 1990; Merchán et al., 2005). The presence of these excitatory and inhibitory transmitters suggests extensive interactions and modulations between neurons in one IC, because excitation and inhibition are the two most important neural interactions that modulate auditory 
signal processing by increasing and decreasing responses of auditory neurons. To study the effect of neural interactions on sound amplitude and frequency selectivities of IC neurons, the auditory responses including the rate-intensity function (RIF) and frequency tuning curve (FTC) of each IC neuron in two simultaneously recorded IC neurons (or paired neurons) were examined under two-tone stimulation conditions. A pair of electrodes was used to simultaneously record two IC neurons in the same isofrequency lamina or different iso-frequency (non-iso-frequency) laminae of the IC (Figure 1). A modulating tone with the best frequency $(\mathrm{BF})$ of one of the paired IC neurons was delivered prior to a probe tone. This two-tone stimulating paradigm provided an opportunity to examine how a neuron activated by its BF sound might affect the response of the other neuron in amplitude and frequency domains. In particular, this procedure allows us to study the possible correlation of each pair of neurons in signal processing. For example, when a pair of IC neurons was stimulated by their two BF tones, the response of one IC neuron was either inhibited (two-tone suppression, Figure 1A) or facilitated (two-tone facilitation, Figure 1B) by the other. It has been reported that the proportion of neurons inhibited by interactions between simultaneously recorded neurons was always higher than that of facilitated neurons (Jen et al., 2002; Wu and Jen, 2008). Thus, the high level of inhibition in IC is basically similar to that in other reports (Vater et al., 1992; Suga, 1995; Fuzessery and Hall, 1996; Zhou and Jen, 2000; Lu and Jen, 2002; Mayko et al., 2012).

Further testing of inhibitory interactions on responses of the paired neurons revealed that the percent two-tone suppression of auditory responses decreased significantly with BF and recording depth differences between paired IC neurons (Jen et al., 2002). This observation is similar to a study in which auditory spatial selectivity of IC neurons was studied under two-tone stimulation conditions (Zhou and Jen, 2000). It was proposed that this phenomenon might be caused by the tonotopic organization of IC neurons, and that inputs from neurons with small BF differences arrive earlier with less attenuation than neurons with large BF differences (Jen et al., 2002). On the other hand, this observation also suggests a gradient of decreasing two-tone suppression along the dorsoventral axis of the IC (Schreiner and Langner, 1997). However, the neural basis underlying this observation remains to be explored.

Because the two-tone stimulation was based on the BFs of two simultaneously recorded neurons, two-tone suppression and facilitation might be thought to be caused by interactions between the two simultaneously recorded neurons activated by their respective BF sounds. Since IC neurons are tonotopically organized, interactions between the IC neurons are actually interactions between frequency laminae or bands. For a pair of IC neurons simultaneously recorded in big brown bat, a sound with the BF of one neuron could modulate the frequency tuning of another neuron by sharpening or broadening it's FTC (Wu et al., 2004). The pairs of neurons involved in frequency band interaction are not only within the same frequency band, but also across different frequency bands. The sharpening degrees of neurons within the same frequency band are higher than those of neurons across frequency bands. It was also found that the strength of frequency band interactions was weaker near the BF but gradually increased with frequency away from the BF of FTC (Wu et al., 2004). Moreover, FTCs of neurons with a BF of $20-30 \mathrm{kHz}$ are most strongly sharpened which is similar to that observed in the chinchilla (Biebel and Langner, 2002).

These data suggest that IC neurons are highly correlated during frequency analysis such that frequency selectivity of the IC neurons is improved through inhibition while the spectrum of frequency sensitivity of other IC neurons is enhanced through excitation.

To further explore the mechanism underlying the effect of two-tone suppression on the responses of two simultaneously recorded neurons, bicuculline (an antagonist of $\mathrm{GABA}_{\mathrm{A}}$ receptor) was applied to one of the paired IC neurons in big brown bat to abolish GABAergic inhibition (Figure 2). Using a pair of neurons (A and $B$, for example), when bicuculline was applied to neuron A, it's number of impulses was greatly increased (Figure 2 Aa vs Aa+bic), and the two-tone suppression was completely removed in neuron A (Figure $2 \mathrm{Aa}+\mathrm{bic}$ vs $\mathrm{Aa}+\mathrm{bic}$ $\vdash \mathrm{b}$ ), but was stronger in neuron $\mathrm{B}$ (Figure $2 \mathrm{Bb} \vdash$ a vs $\mathrm{Bb}$ $\vdash$ a+bic). Thus, the degree of response inhibition decreased in the bicuculline-applied neuron but increased in the paired neuron, suggesting that GABAergic inhibition directly mediated the inhibitory interactions between two simultaneously recorded or paired IC neurons (Wu and Jen, 2008, 2009). However, for another pair of neurons $\mathrm{C}$ and $\mathrm{D}$, the number of impulses greatly increased following bicuculline administration to neuron $\mathrm{C}$ (Figure $2 \mathrm{Cc}$ vs $\mathrm{Cc}+\mathrm{bic}$ ), but the two-tone suppression was only partly abolished in neuron $\mathrm{C}$ (Figure $2 \mathrm{Cc}+$ bic vs $\mathrm{Cc}+$ bic $\mathrm{d}$ ), and was slightly increased in neuron D (Figure $2 \mathrm{Dd} \vdash$ a vs $\mathrm{Dd} \vdash \mathrm{c}+$ bic $)$. A previous study in big brown bat indicated that IC neurons with $\mathrm{GABA}_{\mathrm{A}}$ receptors are mostly distributed in the dorsomedial region but are sparsely distributed in the ventrolateral region which is mostly distributed with neurons containing glycine receptors (Fubara et al., 1996). Therefore, the degree of GABA-mediated two-tone suppression would progressively decrease along the dorsoventral axis of the IC. In brief, when an IC neuron is excited, it may inhibit other neighboring neurons to stand out as the best in the neurons through inhibitory interaction. These inhibitory interactions between neurons in one IC improves auditory sensitivity during auditory signal processing.

\section{BILATERAL COLLICULAR INTERACTION IN AUDITORY SIGNAL PROCESSING}

Many previous studies have clearly shown the anatomical connections between two ICs through the commissure of IC (CoIC). Injecting retrograde tracer in one IC demonstrated that commissure neurons in the central nucleus of IC (ICc) sent projections or fibers to the central nucleus, dorsal and lateral cortices of opposite IC. The commissural fibers ending in the contralateral IC to the injection point formed a laminar plexus that was symmetrical to the ipsilateral plexus, and interconnected mirror symmetric regions of the ICs representing similar frequency bands (Saldaña and Merchán, 1992; Malmierca etal., 1995). Even in the ICc, retrograde labeling of neurons demonstrated that commissural neurons send a divergent projection to the whole extent of the 


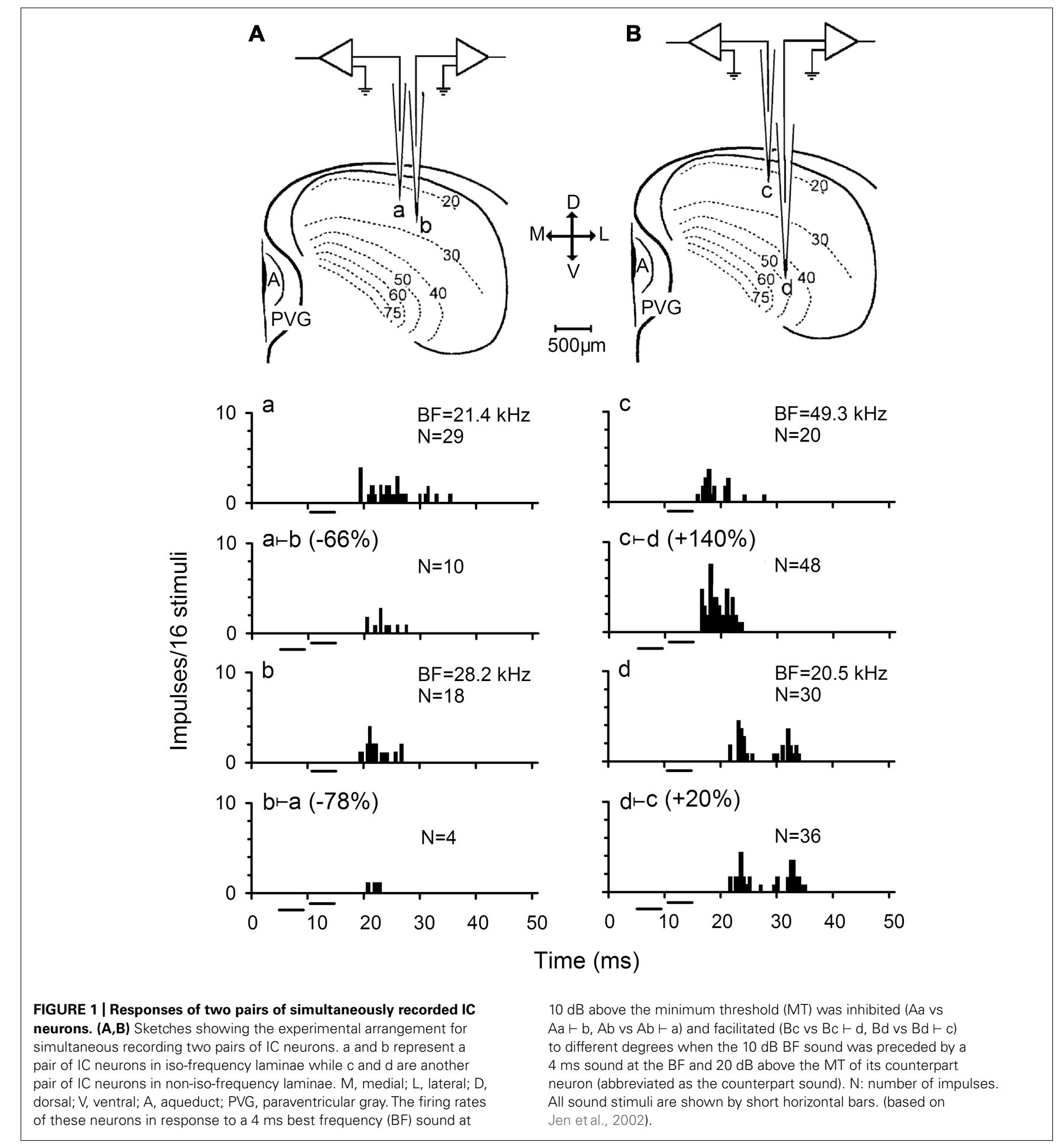

contralateral lamina, which resulted in a V-shaped axonal plexus that covered most of the ICc laminae and extended into the dorsal and lateral cortices. However, the density of the labeled commissurally projecting neurons was weighted toward a point that matched the position of the corresponding tracer injection into the contralateral IC, which is consistent with a point-to-point pattern (Figure 3). The coexistence of point-to-point and divergent projections suggest that CoIC is likely to be involved in interactions between specific regions of corresponding frequency band laminae as well as in integration across the laminae. (Malmierca et al., 2009).

An immunocytochemistry study in CoIC (Saint Marie, 1996) demonstrated the presence of both excitatory projections mediated by glutamate and inhibitory projections mediated by 


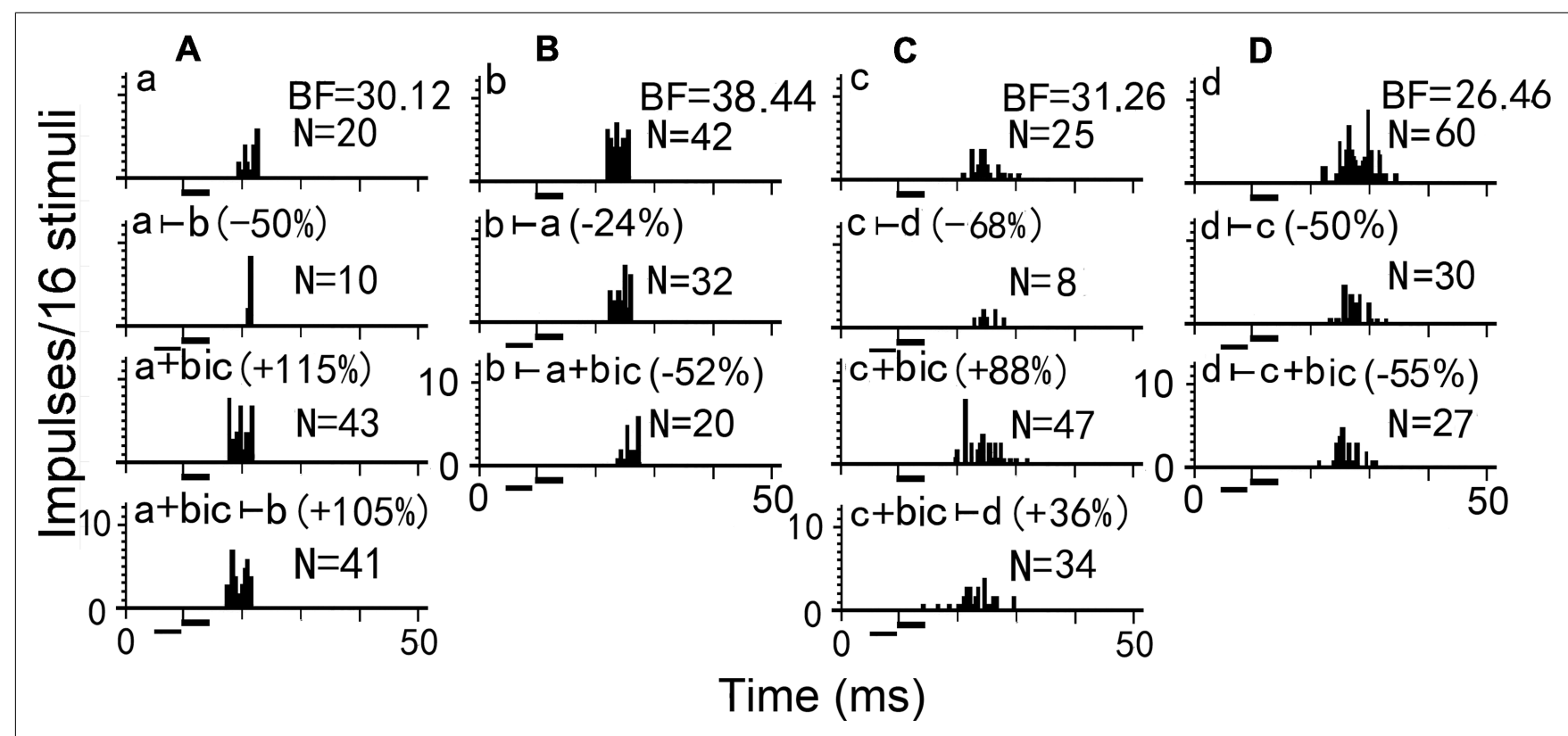

FIGURE 2 | Two-tone suppression on responses of two pairs of simultaneously recorded IC neurons before and during bicuculline (bic) application. Presentation of a counterpart sound decreased the firing rates of each IC neuron in the (A,B) pair or (C,D) pair (Aa, Bb, Cc, Dd vs Aa $\vdash$ b, Bb $\vdash \mathrm{a}, \mathrm{Cc} \vdash \mathrm{d}$, Dd $\vdash \mathrm{c}$ ). When bicuculline was applied to neurons $A$ and $C$, the number of impulses increased (Aa vs $\mathrm{Aa}+$ bic, $\mathrm{Cc}$ vs $\mathrm{Cc}+\mathrm{bic}$ ). The presentation of a counterpart sound during bicuculline application to neurons $A$ and $C$ decreased the number of impulses only slightly in neuron $A$ but substantially in neuron $\mathrm{C}$ (Aa+bic vs $\mathrm{Aa}+$ bic $\vdash$ b, $\mathrm{C} c+$ bic vs $\mathrm{C} c+$ bic $\vdash \mathrm{d})$, however, decreased the number of impulses substantially in neuron $B$ and slightly in neuron $\mathrm{D}$ (Bb vs $\mathrm{Aa}+\mathrm{bic} \vdash \mathrm{b}, \mathrm{C} c+$ bic vs $\mathrm{C} c+$ bic $\vdash \mathrm{d}$ ). N: number of impulses. All sound stimuli are shown by short horizontal bars. Mu and Jen, 2008)

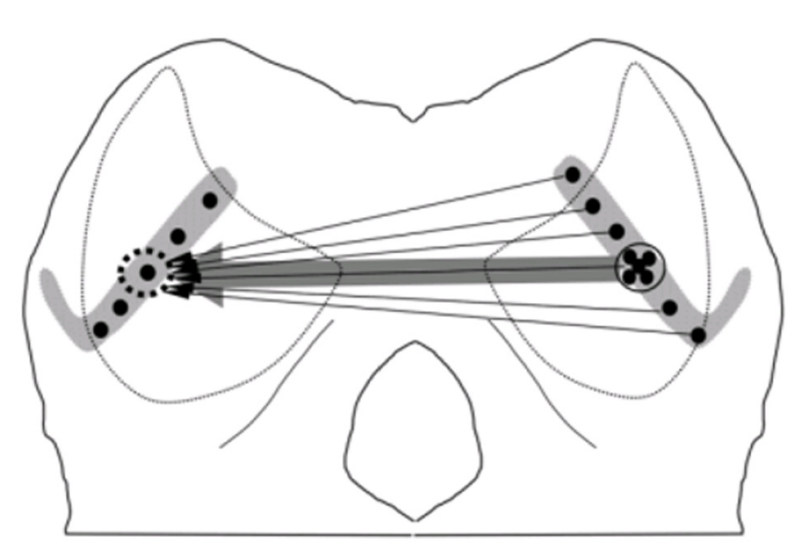

FIGURE 3 | Schematic wiring diagrams of the commissural connections. In the central nucleus of IC (ICC), the retrograde labeling of neurons demonstrated that an injection into one point on the lamina (dotted circle, left IC) retrogradely labeled neurons over the whole extent of the contralateral lamina, consistent with a divergent pattern of connections (thin arrows). The density of the projection is centered on a point matching the position of the tracer injection which is consistent with a

point-to-point-weighted wiring pattern (thick arrow; Malmierca et al., 2009).

GABA. Injections of $\mathrm{D}-[3 \mathrm{H}]$ aspartate which is considered a selective marker for glutamatergic synapses, suggested that some glutamatergic endings in the IC originated from the opposite IC in the chinchilla. Studies that combined tract-tracing with horseradish peroxidase (HRP) and immunocytochemical labeling for GABA, found that double labeled neurons were mostly in the contralateral IC following a tracer injection into the ipsilateral IC in rat. These GABAergic CoIC could exert a direct monosynaptic inhibitory influence on their contralateral counterparts (González-Hernández et al., 1996; Hernández et al., 2006).

These anatomical findings are consistent with an electrophysiological study that concentrated on the interactions between two ICs. In vitro whole cell recording of IC neurons demonstrated that an excitatory and inhibitory postsynaptic current (EPSC and IPSC) was evoked by direct stimulation of the CoIC. The addition of GABAergic or glycinergic antagonists to CoIC could reduce the IPSC to various degrees, even there was a strong inhibitory input that was almost exclusively GABAergic. Furthermore, ionotropic glutamic receptor antagonists reduced both the EPSC and IPSC. This indicated that much of the inhibitory input appears to be mediated by interneuronal connection (Moore et al., 1998). Inactivation of excitatory CoIC could inhibit recorded IC neurons by direct elimination of the excitation and facilitate recorded neurons by disinhibiting inhibitory synapse of interneurons.

Bilateral collicular interaction between two ICs in auditory signal processing were examined using extracellular recordings in vivo. Malmierca et al. $(2003,2005)$ blocked the transmission of excitatory fibers in CoIC by means of local hydraulic injection of kynurenic acid (KA; a non-specific glutamatergic receptor antagonist) into one IC and observed changes in the frequency response area, number of impulses and monotonicity of neurons located in the corresponding region of the contralateral IC. 


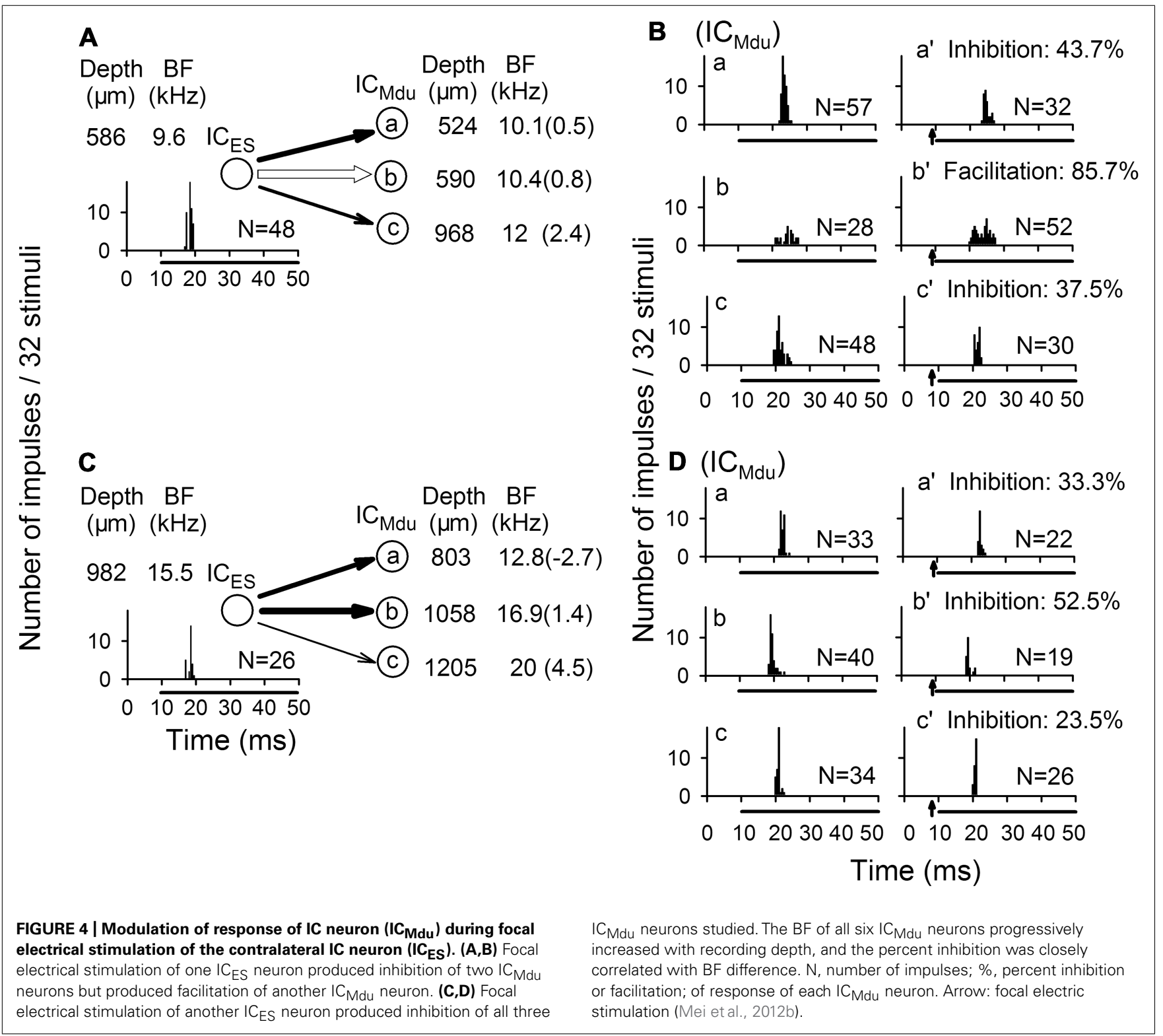

These studies indicated bilateral collicular interactions in the corresponding frequency laminae between the two ICs that were mediated by CoIC. Consistent with the result of whole cell recording, focal injection of KA in one IC both decreased and increased the number of impulses in the opposite IC neurons. This provided further evidence for an inhibitory influence mediated by inhibitory interneuronal connection.

Moreover, our recent study also demonstrated that focal electrical stimulation of one IC produced widespread inhibition and focused excitation of responses in contralateral IC neurons. The excitatory modulation of bilateral collicular interactions expands the RIFs and FTCs of facilitated IC neurons but decreased the slope of their RIFs and $\mathrm{Q}_{10}$ value of their FTCs for wider amplitude and frequency responses to sound stimuli. Conversely, the inhibitory modulation of bilateral collicular interaction sharpens the RIFs and FTCs of inhibited IC neurons but increased the slope of their RIFs and $\mathrm{Q}_{10}$ value of their FTCs for sharper sensitivity to sound amplitude and frequency (Mei et al., 2012b; Cheng et al., 2013). It is also suggested that the small proportion of bilateral collicular excitatory interactions between neurons in corresponding frequency laminae and the large proportion of bilateral collicular inhibitory interactions between neurons in different frequency laminae may be involved in the formation of binaural neurons (i.e., excitation-excitation, EE neurons that can be excited by same BF sound stimulation to either ear; excitation-inhibition, EI neurons that are strongly excited by sound stimulation to the contralateral ear and are inhibited by sound stimulation to the ipsilateral ear; Mei et al., 2012a). The possible neural pathway may be described that the excitation from ipsilateral ear can cross to the contralateral IC in a lower auditory nucleus and then to the ipsilateral IC via facilitatory or inhibitory CoIC, respectively. The unbalanced properties between excitatory and inhibitory projections have a very 
important role in the formation of unilateral auditory dominance and sound location.

In accordance with the anatomical data of point-to-point and divergent projections between two ICs, focal electrical stimulation of one neuron modulated the responses of three contralateral neurons (Figures $4 \mathrm{~A}$ vs $4 \mathrm{~B}$ and $4 \mathrm{C}$ vs $4 \mathrm{D}$ ). Each of three contralateral IC neurons was sequentially isolated at a different depth with a progressive increase in BF. The degree of bilateral collicular interaction was dependent upon the BF difference between the electrically stimulated IC neurons and the modulated IC neurons. The percent modulation in the number of impulses was larger for the neuron with a smaller BF difference than for the neuron with a larger BF difference (Mei et al., 2012b).

In addition, after the focal electrical stimulation was delivered for $30 \mathrm{~min}$, a long term shift in an IC neuron's BF was induced which remained for as long as $150 \mathrm{~min}$ and decreased with time (Cheng et al., 2013). Therefore the bilateral collicular interaction modulates both auditory signal processing and auditory plasticity of IC neurons that is similar to the corticofugal modulation of IC neurons (Jen et al., 1998; Ma and Suga, 2001; Suga et al., 2002; Yan and Ehret, 2002; Jen and Zhou, 2003; Yan et al., 2005; Zhou and Jen, 2007). Since the BF-dependent modulation of bilateral collicular interaction is not entirely comparable to the egocentric selectivity of corticofugal modulation, further studies are required to determine whether the modulation effect of bilateral collicular interactions might also be mediated through corticofugal feedback loops.

Interestingly, following reciprocal electrical stimulation of pairs of neurons, respectively, in two ICs, we found that the bilateral collicular interaction was either reciprocal or unilateral. However, after HRP deposits were made in CoIC, regions of the IC supplying fibers to the commissure were not the main targets for the

\section{REFERENCES}

Adams, J. C. (1979). Ascending projections to the inferior colliculus. J. Comp. Neurol. 183, 519-538.

Adams, J. C., and Mugnaini, E. (1984). Dorsal nucleus of the lateral lemniscus: a nucleus of GABAergic projection neurons. Brain Res. Bull. 13, 585-590.

Adams, J. C., and Wenthold, R. (1979). Distribution of putative amino acid transmitters, choline acetyltransferase and glutamate decarboxylase in the inferior colliculus. Neuroscience 4 , 1947-1951.

Aitkin, L. M., and Phillips, S. C. (1984). The interconnections of the inferior colliculi through their commissure. J. Comp. Neurol. 228, 210-216.

Biebel, U. W., and Langner, G. (2002). Evidence for interaction across frequency channels in the inferior colliculus of awake chinchilla. Hear. Res. $169,151-168$.

Brunso-Bechtold, J. K., Thompson, G. C., and Masterton, R. B. (1981). HRP study of the organization of auditory afferents ascending to central nucleus of inferior colliculus in cat. J. Comp. Neurol. 197, 705-722.

Casseday, J. H., and Covey, E. (1996). A neuroethological theory of the operation of the inferior colliculus. Brain Behav. Evol. 47, 311-336.

Caspary, D. M., Raza, A., Lawhorn Armour, B. A., Pippin, J., and Americ, S. P. (1990). Immunocytochemical and neurochemical evidence for agerelated loss of GABA in the inferior colliculus: implications for neural presbycusis. J. Neurosci. 10, 2363 2372.

Casseday, J. H., Ehrlich, D., and Covey, E. (1994). Neural tuning for sound duration: role of inhibitory mechanisms in the inferior colliculus. Science 264, 847-850.

Cheng, L., Mei, H. X., Tang, J., Fu, Z. Y., Jen, P. H. S., and Chen, Q. C. (2013). Bilateral collicular interaction: modulation of auditory signal processing in frequency domain. Neuroscience 235, 27-39.

terminals of these fibers, which suggested that interconnections of the ICs through their commissure were complementary, rather than reciprocal (Aitkin and Phillips, 1984).

\section{PROSPECTS}

Neural interactions are of great interest because of their contribution to sensory information processing, neural functional integration and neural modulation. As for the auditory midbrain, neural interactions were found both in one IC and between two ICs, even in unilateral iso-frequency and non-iso-frequency laminae as well as bilateral corresponding and non-corresponding frequency laminae. Generally, there is a large percentage of inhibitory interactions but a small percentage of excitatory interactions, which is likely because of the presence of many inhibitory interneurons. These excitatory and inhibitory interactions in or between ICs modulate auditory signal processing in amplitude and frequency domains, and provide an adjustable and plastic modulation pattern for the auditory signal processing of ICs. However, many details, such as neural plasticity of the structure and function as well as cellular and synaptic mechanisms of the neural modulation underlying neural interactions in auditory signal processing, remain unclear and require further study. We have sufficient reasons to believe that new knowledge about the various neural interactions will be obtained with successive studies. Thus, the studies of neural interactions in one IC and between two ICs are in the ascendancy.

\section{ACKNOWLEDGMENTS}

We thank the anonymous reviewers for commenting on an earlier version of this manuscript. This work was supported by the grants (\#31070971, \#30970972) from the National Science Foundation of China.

Covey, E., and Casseday, J. H. (1995). "The lower brainstem auditory pathways," in Springer Handbook of Auditory Research Vol. 5, Hearing by Bats, eds A. N. Popper, and R. R. Fay (New York: Springer), 235-295.

Fubara, B. M., Casseday, J. H., Covey, E., and Schwartz-Bloom, R. D. (1996) Distribution of GABAA, GABAB and glycine receptors in the central auditory system of the big brown bat, Eptesicus fuscus. J. Comp. Neurol. 369, 83-92.

Fuzessery, Z. M., and Hall, J. C. (1996). Role of GABA in shaping frequency tuning and creating FM sweep selectivity in the inferior colliculus. J. Neurophysiol. 76, 1059-1073.

Games, K. D., and Winer, J. A. (1988). Layer $\mathrm{V}$ in rat auditory cortex: projections to the inferior colliculus and contralateral cortex. Hear. Res. 34, $1-25$.

González-Hernández， T., MantolánSarmiento, B., González-González, B., and Pérez-González, H. (1996). Sources of GABAergic input to the inferior colliculus of the rat, J. Comp. Neurol. 372, 309-326.

Herbert, H., Aschoff, A., and Ostwald, J. (1991). Topography of projections from the auditory cortex to the inferior colliculus in the rat. J. Comp. Neurol. 304, 103-122.

Hernández, O., Rees, A., and Malmierca, M. S. (2006). A GABAergic component in the commissure of the inferior colliculus in rat. Neuroreport 17 , 1611-1614.

Herrera, M., Correa, J., Sanchez del Campo, F., and Ruiz, A. (1988). Stellate cells and their axonal patterns in the central nucleus of the inferior colliculus of the cat (Felis domesticus). J. Hirnforsch. 29, 393-402.

Herrera, M., Shchez del Campo, F., Puchades, A., and Correa, J. (1989). Axonal patterns of discshaped cells in the central nucleus of the cat inferior colliculus. Z. mikrosk. Anat. Forsch. $103,515-525$.

Jen, P. H. S., Chen, Q. C., and Sun, X. D. (1998). Corticofugal regulation of auditory sensitivity in the bat inferior 
colliculus. J. Comp. Physiol. A 183, 683-697.

Jen, P. H. S., Wu, F. J., and Chen, Q. C. (2002). The effect of two-tone stimulation on responses of two simultaneously recorded neurons in the inferior colliculus of the big brown bat, Eptesicus fuscus. Hear. Res. 168, 139-149.

Jen, P. H. S., and Zhang, J. P. (2000). The role of GABAergic inhibition on direction-dependent sharpening of frequency tuning in bat inferior collicular neurons. Brain Res. 862, 127-137.

Jen, P. H. S., and Zhou, X. M. (2003). Corticofugal modulation of amplitude domain processing in the midbrain of the big brown bat, Eptesicus fuscus. Hear. Res. 184, 91-106.

LeBeau, F. E., Malmierca, M. S., and Rees, A. (2001). Iontophoresis in vivo demonstrates a key role for GABAA and glycinergic inhibition in shaping frequency response areas in the inferior colliculus of guinea pig. $J$. Neurosci. 21, 7303-7312.

Lu, Y., and Jen, P. H. S. (2002). Interaction of excitation and inhibition in inferior collicular neurons of the big brown bat, Eptesicus fuscus. Hear. Res. 169, 140-150.

Ma, X., and Suga, N. (2001). Plasticity of bat's central auditory system evoked by focal electric stimulation of auditory and/or somatosensory cortices. J. Neurophysiol. 85, 1078-1087.

Ma, X., and Suga, N. (2008). Modulation of the paradoxical latency shifts of inferior collicular neurons. J. Neurophysiol. 100, 1127-1134.

Malmierca, M. S., Hernández, O., Antunes, F. M., and Rees, A. (2009). Divergent and point-to-point connections in the commissural pathways between the inferior colliculi. $J$. Comp. Neurol. 514, 226-239.

Malmierca, M. S., Hernandez, O., Falconi, A., Lopez-Poveda, E. A., Merchan, M., and Rees, A. (2003). The commissure of the inferior colliculus shapes frequency response areas in rat: an in vivo study using reversible blockade with microinjection of kynurenic acid. Exp. Brain Res. 153, 522-529.

Malmierca, M. S., Hernandez, O., and Rees, A. (2005). Intercollicular commissural projections modulate neuronal responses in the inferior colliculus. Eur. J. Neurosci. 21, 27012710.

Malmierca, M. S., Rees, A., Le Beau, F. E., and Bjaalie, J. G. (1995). Laminar organization of frequency-defined local axons within and between the inferior colliculi of the guinea pig. J. Comp. Neurol. 357 124-144.

Malmierca, M. S., and Ryugo, D. K. (2011). "Descending connections of auditory cortex to the midbrain and brain stem," in The Auditory Cortex, eds J. Winer, and C. E. Schreiner (New York: Springer) 189-208.

Malmierca, M. S., Saint Marie, R. L. Merchan, M. A., and Oliver, D. L. (2005). Laminar inputs from dorsal cochlear nucleus and ventral cochlear nucleus to the central nucleus of the inferior colliculus: two patterns of convergence. Neuroscience 136, 883-894.

Mayko, Z. M., Roberts, P. D., and Portfors, C. V. (2012). Inhibition shapes selectivity to vocalization in the inferior colliculus of awake mice. Front. Neural Circuits 6:73. doi: 10.3389/fncir.2012.00073

Merchán, M., Aguilar, L. A., LopezPoveda, E. A., and Malmierca, M. S. (2005). The inferior colliculus of the rat: quantitative immunocytochemical study of GABA and glycine. Neuroscience 136, 907-925.

Mei, H. X., and Chen, Q. C. (2010). Neural modulation in inferior colliculus and central auditory plasticity. Front. Biol. 5, 123-127.

Mei, H. X., Cheng, L., Tang, J., Fu, Z. Y., Jen, P. H. S., and Chen, Q. C. (2012a). Modulation of amplitude sensitivity by bilateral collicular interaction among different frequency laminae. Neurosci. Lett. 517, 13-17.

Mei, H. X., Cheng, L., Tang, J., Fu, Z. Y., Jen, P. H. S., and Chen, Q.-C (2012b). Bilateral inferior collicular interaction: modulation of auditory signal processing in amplitude domain. PLoS ONE 7:e41311. doi: 10.1371/journal.pone.0041311

Moore, D. R., Kotak, V. C., and Sanes D. H. (1998). Commissural and lemniscal synaptic input to the gerbil inferior colliculus. J. Neurophysiol. 80, 2229-2236.

Moore, J. K., and Moore, R. Y. (1987). Glutamic acid decarboxylase-like immunoreactivity in brainstem auditory nuclei of the rat. J. Comp. Neurol. 260, 157-174.

Ojima, H. (1994). Terminal morphology and distribution of corticothalamic fibers originating from layers 5 and 6 of cat primary auditory cortex. Cereb. Cortex 4 646-663.
Oliver, D. L., and Schneiderman, A. (1991). "The anatomy of the inferior colliculus: A cellular basis for integration of monaural and binaural information," in Neurophysiology of Hearing: The central auditory system, eds R. A. Altschuler, R. P. Bobbin, B. M. Clopton, and D. W. Hoffman (New York: Raven Press), 195-222.

Ottersen, O. P., and Storm-Mathisen, J. (1984). "Neurons containing or accumulating transmitter amino acids," in Handbook of Chemical Neuroanatomy, Vol. 3, Classical Transmitters and Transmitter Receptors in the CNS. eds A. Bjorklund, T. Hokfelt, and M.J. Kuhar (Amsterdam: Elsevier), 141-246.

Pollak, G. D., and Casseday, J. H. (eds). (1989). The Neural Basis of Echolocation in Bats. Berlin: Springer press.

Popelar, J., Nwabueze-Ogbo, F. C., and Syka, J. (2003). Changes in neuronal activity of the inferior colliculus in rat after temporal inactivation of the auditory cortex. Physiol. Res. 52, 615-628.

Roberts, R. C., and Ribak, C. E. (1987). An electron microscopic study of GABAergic neurons and axon terminals in the central nucleus of the inferior colliculus of the rat. J. Neurocytol. 16, 333-345.

Saint Marie, R. L. (1996). Glutamatergic connections of the auditory midbrain: selective uptake and axonal transport of $\mathrm{D}$-[ $[3 \mathrm{H}]$ aspartate. J. Comp. Neurol. 373, 225-270.

Saldaña, E., Feliciano, M., and Mugnaini, E. (1996). Distribution of descending projections from primary auditory neocortex to inferior colliculus mimics the topography of intracollicular projections. J. Comp. Neurol. 371, 15-40.

Saldaña, E., and Merchán, M. A. (1992). Intrinsic and commissural connections of the rat inferior colliculus. J. Comp. Neurol. 319, 417-437.

Schreiner, C. E., and Langner, G. (1997). Laminar fine structure of frequency organization in auditory midbrain. Nature 388, 383-386.

Suga, N. (1995). Sharpening of frequency tuning by inhibition in the central auditory system: tribute to Yasuji Katsuki. Neurosci. Res. 21, 287-299.

Suga, N. (1997). "Parallel-hierarchical processing of complex sounds for specialized auditory function," in Encyclopedia of Acoustics, ed. M. J. Crocker (New York: Wiley), 1409_ 1418.
Suga, N. (2008). Role of corticofugal feedback in hearing. J. Comp. Physiol A 194,169-183.

Suga, N., Ji, W., Ma, X., Tang, J., Xiao, Z., and Yan, J. (2010). "Corticofugal modulation and beyond for auditory signal processing and plasticity," in Auditory and Vestibular Efferents, eds D. K. Ryugo, R. R. Fay, and A. N. Popper, (Berlin: Springer), 313-352.

Suga, N., Xiao, Z., Ma, X., and Ji, W. (2002). Plasticity and corticofugal modulation for hearing in adult animals. Neuron 36, 9-18.

Suga, N., Yan, J., and Zhang, Y. F. (1998). "The processing of species-specific complex sounds by the ascending and descending auditory systems," in Central Auditory Processing and Neural Modeling, eds P. Poon, and J. Brugge (New York: Plenum Press), 55-70.

Vater, M., Habbicht, H., Kossl, M., and Grothe, B. (1992). The functional role of GABA and glycine in monaural and binaural processing in the inferior colliculus of horseshoe bats. J. Comp. Physiol. A 171, 541-553.

Vetter, D. E., and Mugnaini, E. (1984). Immunocytochemical localization of GABAergic elements in the rat inferior colliculus. Soc. Neurosci. Abstr. $10,1148$.

Wu, F. J., Chen, Q. C., Jen, P. H. S., and Shen, J. X. (2004). Effect of frequency-band integration on the sharpening frequency tuning of IC neurons in bat, Eptesicus fuscus. Chin. Sci. Bull. 49, 1026-1031.

Wu, F. J., and Jen, P. H. S. (2008). GABA-mediated modulation of the discharge pattern and rate-level function of two simultaneously recorded neurons in the inferior colliculus of the big brown bat, Eptesicus Fuscus. Chin. J. Physiol. 51, 13-26.

Wu, F. J., and Jen, P. H. S. (2009). Involvement of GABAmediated inhibition in shaping the frequency selectivity of neurons in the inferior colliculus of the big brown bat, Eptesicus fuscus. Chin. J. Physiol. 52, 1-9.

Yan, J., and Ehret, G. (2002). Corticofugal modulation of midbrain sound processing in the house mouse. Eur. J. Neurosci. 16, 119-128.

Yan, J., Zhang, Y., and Ehret, G. (2005). Corticofugal shaping of frequency tuning curves in the central nucleus of the inferior colliculus of mice. J. Neurophysiol. 93, 71-83. 
Zhou, X. M., and Jen, P. H. S. (2000). Neural inhibition sharpens auditory spatial sensitivity of bat inferior collicular neurons. J. Comp. Physiol. A 186, 389-398.

Zhou, X. M., and Jen, P. H. S. (2007). Corticofugal modulation of multi-parametric auditory selectivity in the midbrain of the big brown bats. J. Neurophysiol. 98, 2509-2516.

Conflict of Interest Statement: The authors declare that the research was conducted in the absence of any commercial or financial relationships that could be construed as a potential conflict of interest.
Received: 01 July 2012; accepted: 30 March 2013; published online: 19 Apri 2013.

Citation: Mei H-X, Cheng $L$ and Chen Q-C (2013) Neural interactions in unilateral colliculus and between bilateral colliculi modulate auditory signal processing. Front. Neural Circuits 7:68. doi 10.3389/fncir.2013.00068
Copyright (c) 2013 Mei, Cheng and Chen. This is an open-access article distributed under the terms of the Creative Commons Attribution License, which permits use, distribution and reproduction in other forums, provided the original authors and source are credited and subject to any copyright notices concerning any third-party graphics etc. 